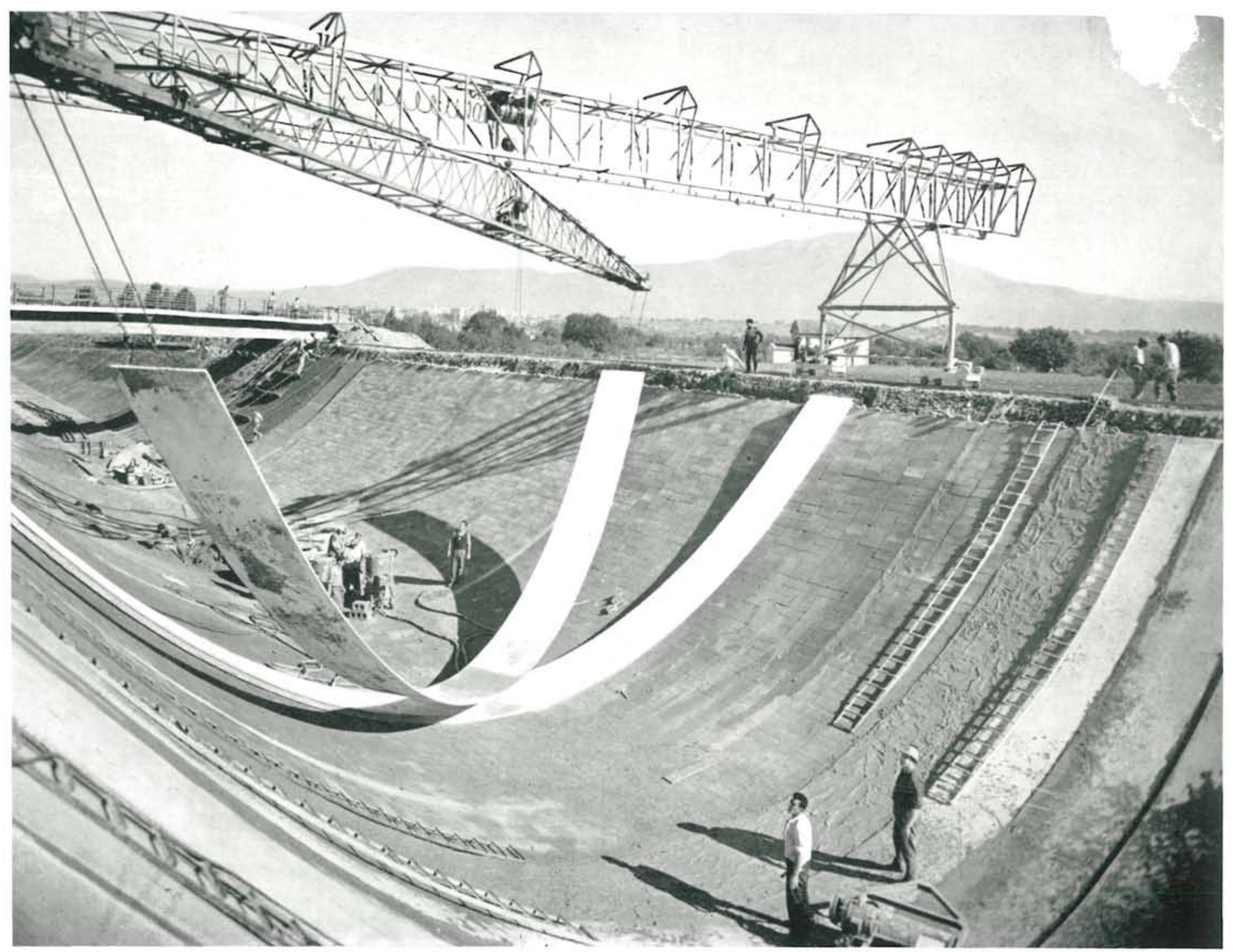

\title{
revestimientos especiales para diques y canales
}

\author{
SILVANO ZORZI, Dr. ingeniero
}

$533 \cdot 20$

Sînoposis En este trabajo se resumen las posibilidades y ventajas que ofrecen los revestimientos especiales de diques y taludes de canales y, en general, en todas las grandes obras hidraulicas, en que la impermeabilzación es de mayor importancia al tratar de conservar los caudales iniciales 0 la re tención de aguas embalsadas, según los casos. Los progresos de las técnicas modernas, en particular la prefabricación, han permitido este tipo de revestimientos con losas de gran longitud, extremadamente flexibles e impermeables, de hormigon armado y pretensado. Aunque son muchas las posibilidades $y$ tipos de revestimiento, destaca el que a continuación se expone: consiste en placas o losas estrechas, de gran flexibiliad, colocadas transversalmente al eje del canal sobre tongadas de morter de cemento, y cuyas juntas se sellan, hín tentan la ciones que constituyen por sí mismas, las conclusiones que del método se pueden sacar para su mejor éxito y explotación, tanto desde el punto de vista téenico como económico. 


\section{Consideraciones generales}

Los revestimientos de diques, azudes y canales tienen por función principal consolidar el perfil mojado y protegerle contra la erosión por el agua provocada. Complementan esta importante característica el mejoramiento del coeficiente de rugosidad o asperezas en lo que respecta al aumento del gasto o caudal, y constitutuye un manto de impermeabilización.

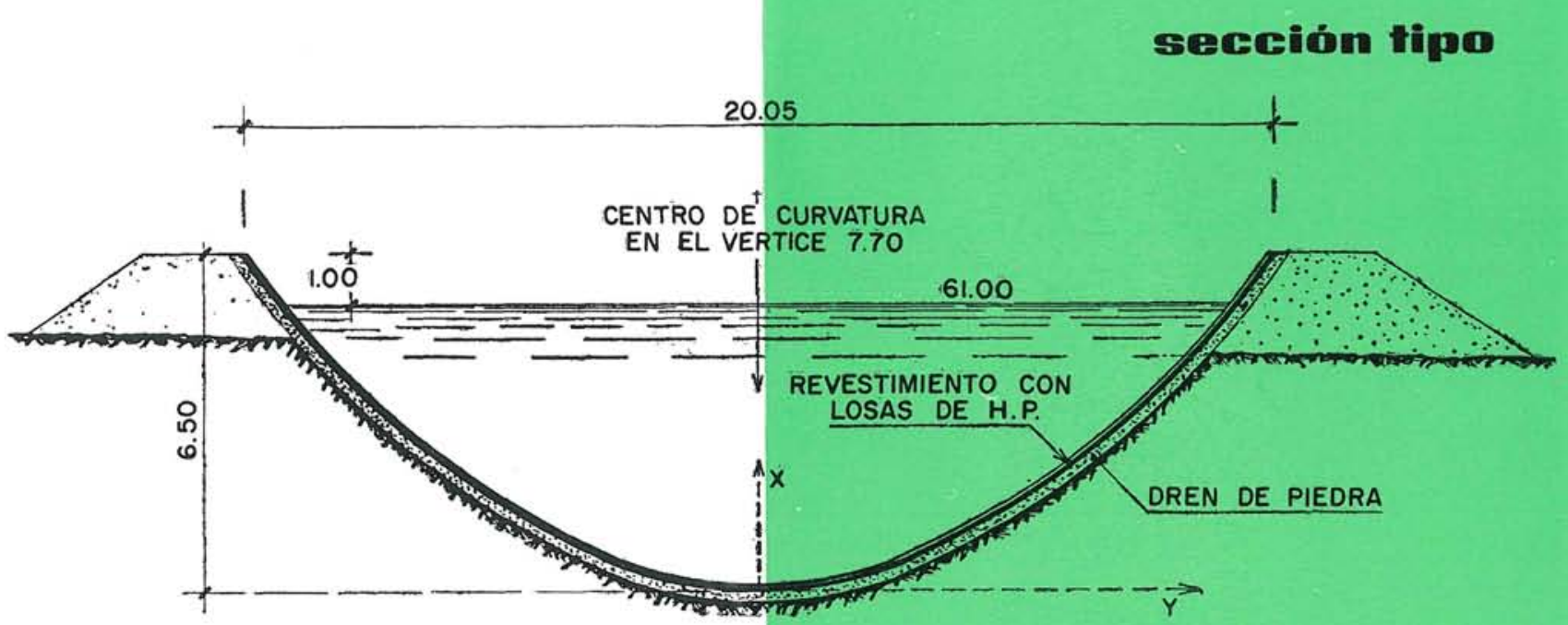

Esta impermeabilización adquiere caracteres inquietantes en los aprovechamientos hidroeléctricos y canaes, principalmente en los de irrigación, donde las fugas son a veces cuantiosas. Tiene también particular interés les, principalmente en los de irrigacion, dón en las capas del revestimiento, ya que éstas se hallan expuestas, con ello, a perder su propia estabilidad.

Los revestimientos presentan diversas características, que, genéricamente, dan origen a las siguientes consideraciones:

a) Los revestimientos de hormigón en masa o armado, colocado en obra, van cediendo el paso a otros tipos de menor rigidez y aún más económicos. A los realizados hormigonando en obra se han opuesto solidación, posibilidad de agrietamiento por retracción, la presencia de asientos locales del terreno y, con ello, propensión a un estado de fragilidad y, por último, la dificultad de una eventual reparación.

b) Dentro del campo de la pavimentación, se prefiere recurrir a la prefabricación de losas que se colocan en obra sobre un lecho adecuadamente preparado. Este último sistema tiene la ventaja de ser más flexible y, consiguientemente, se presta mejor a seguir las deformaciones accidentales del plano de apoyo.

c) Más complejo es el campo de la pavimentación bituminosa, que presenta la característica de un manto continuo adaptable plásticamente al relieve del lecho sobre el que se apoya. Sin embargo, aparecen ciertos problemas aún no resueltos, referentes a la colocación de betunes sobre superficies de inclinación acusada, como son las de los taludes de los canales y diques.

La pavimentación bituminosa es muy sensible al calor (insolación) en la superficie expuesta, así como a las heladas, a las grandes velocidades erosivas del agua y a la esterilización del terreno de apoyo, para impedir que crezca vegetación. Alguno de estos inconvenientes desaparece si se emplean las recientes losas prefabricadas con hormigón armado bituminoso.

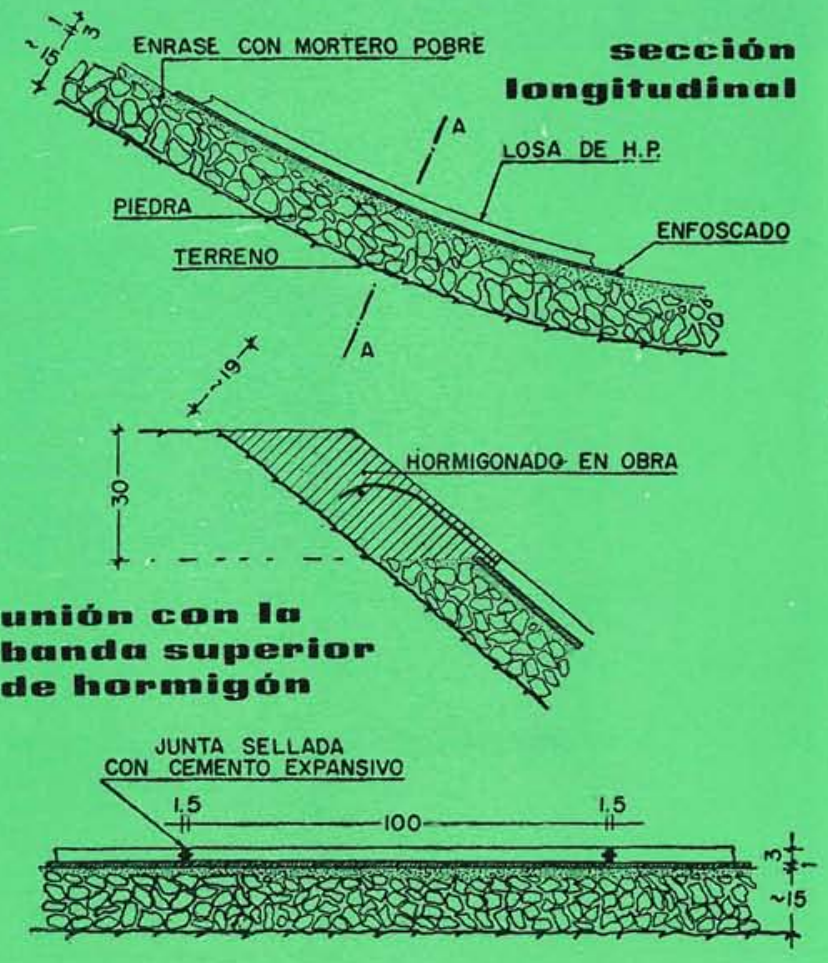

sección trumsuersen $A=A$ 


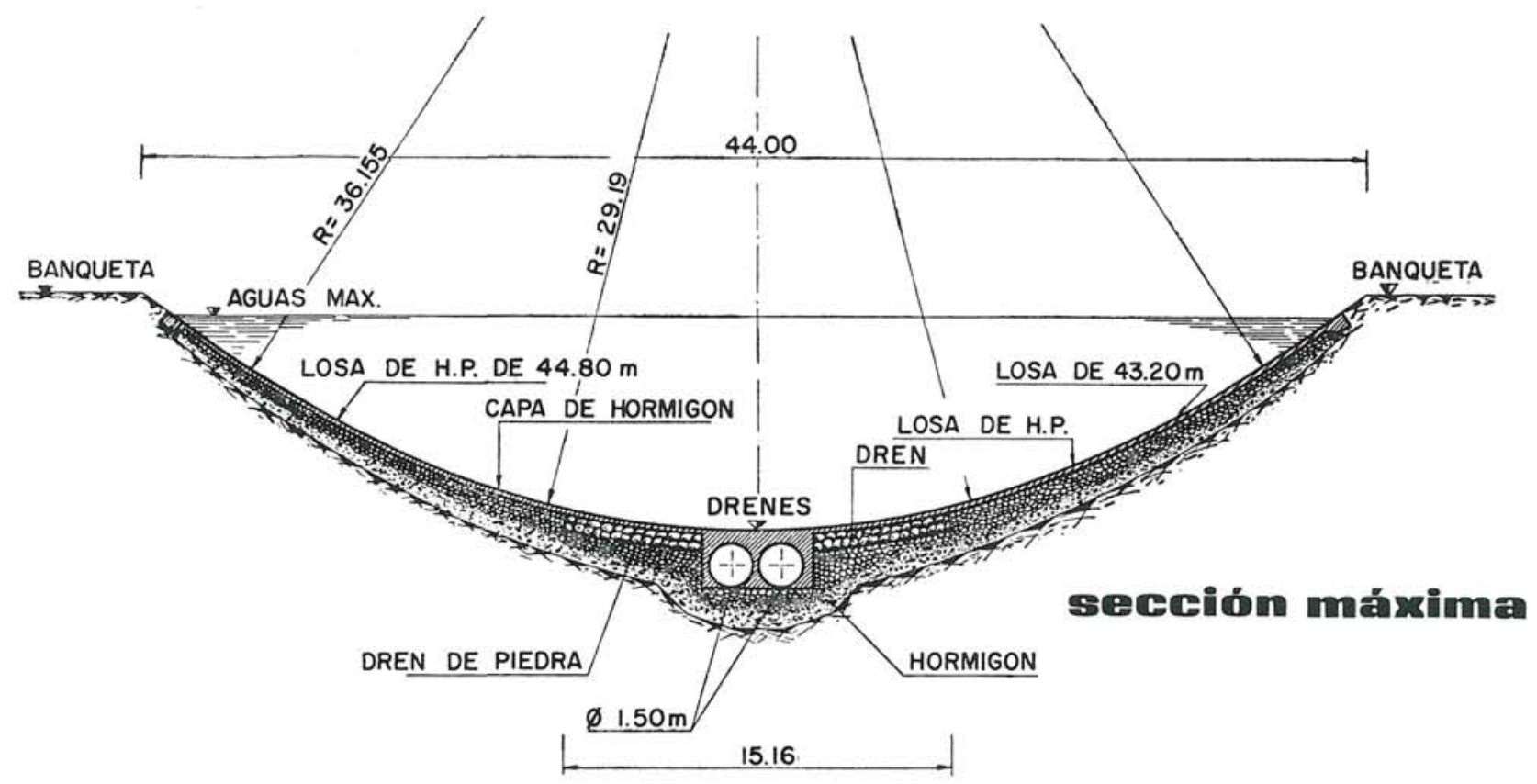

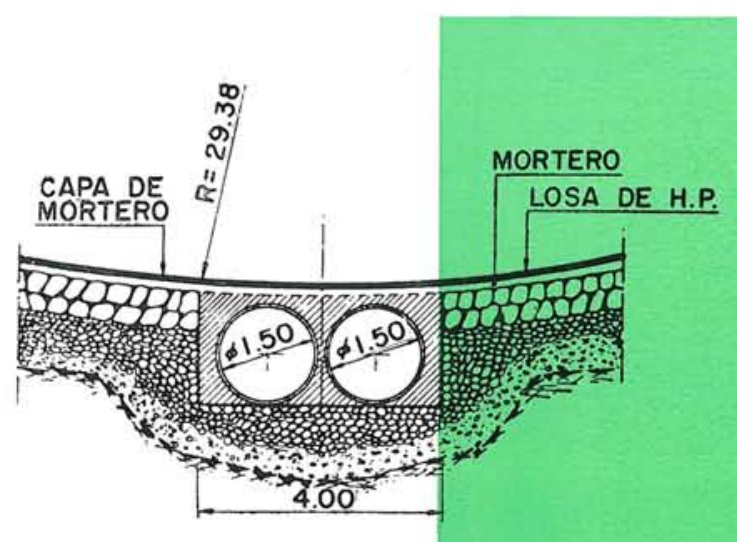

cletralle del fom do

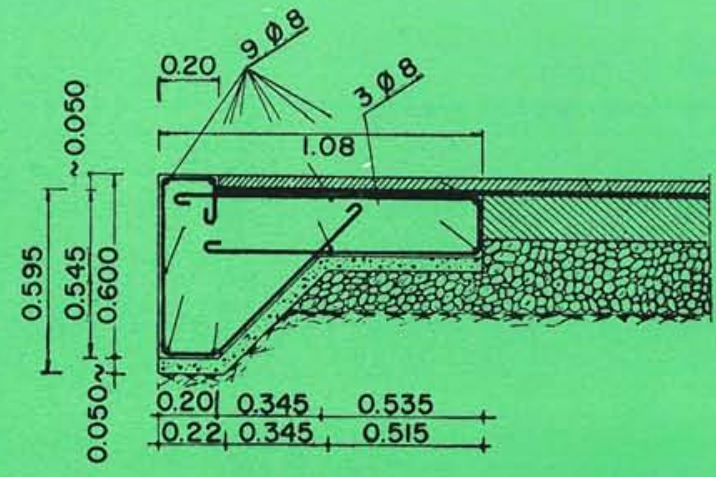

detalle de Ia bangueta

De todo lo dicho se llega a la siguiente conclusión: actualmente la técnica se dirige hacia tipos de pavimentaciones que unan la duración y estabilidad de los materiales del cemento a la plasticidad de los bituminosos.

\section{Losass dle lhormigjón armmado pretrensado}

Desde hace tiempo cree el autor que las técnicas modernas del hormigón armado pretensado podrían encontrar soluciones interesantes en la pavimentación hidráulica, tales como las de los problemas antes apuntados bajo sus dos aspectos: eficiencia y economía.

El hormigón pretensado está especialmente indicado en las obras hidráulicas, ya que la precompresión, convenientemente reglada, puede atenuar la fisuración macro y microscópica. 


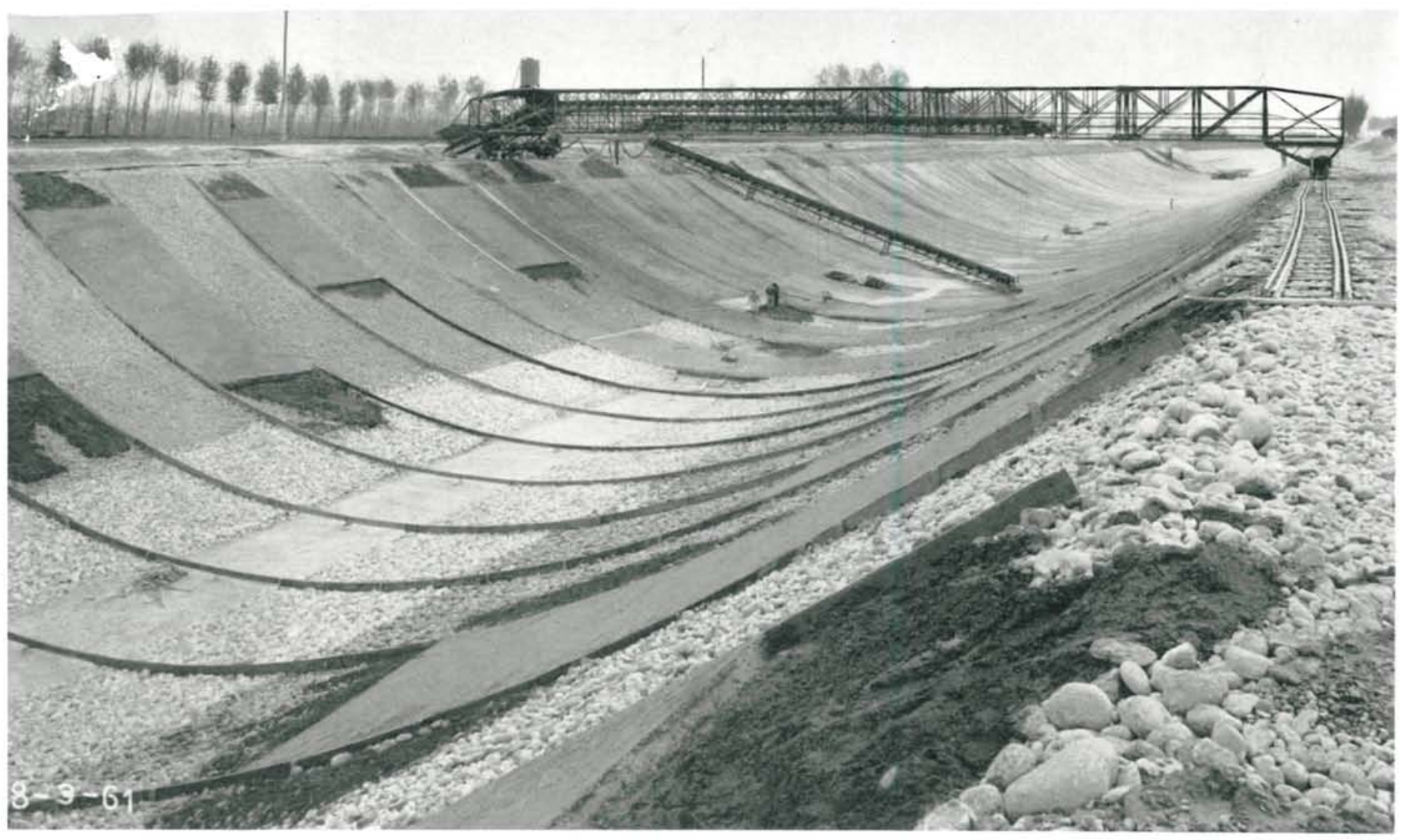

Prerrevestimiento, con hormigón pobre, del canal del Mincio.

Taller de prefabricación de losas.

Por otra parte, el hormigón pretensado se presta favorablemente a la prefabricación, debido a su mayor ligereza y facilidad de colocación y transporte. Dada su adaptabilidad al plano de apoyo, se pensó en la posibilidad de reducir el es pesor al mínimo y obtener una especie de "membrana" sin apreciable rigidez flexional, es decir, una verdadera alfombra tenaz, de hormigón pretensado.

Las características de estas llamadas alfombras o losas ligeras son:

1) Espesores mínimos de 3,4 y $5 \mathrm{~cm}$, compatibles con una capa que cubra las armaduras.

2) Armaduras metálicas baricéntricamente dispuestas.

3) Elección acertada de la granulometría de los áridos y dosificación en cemento.
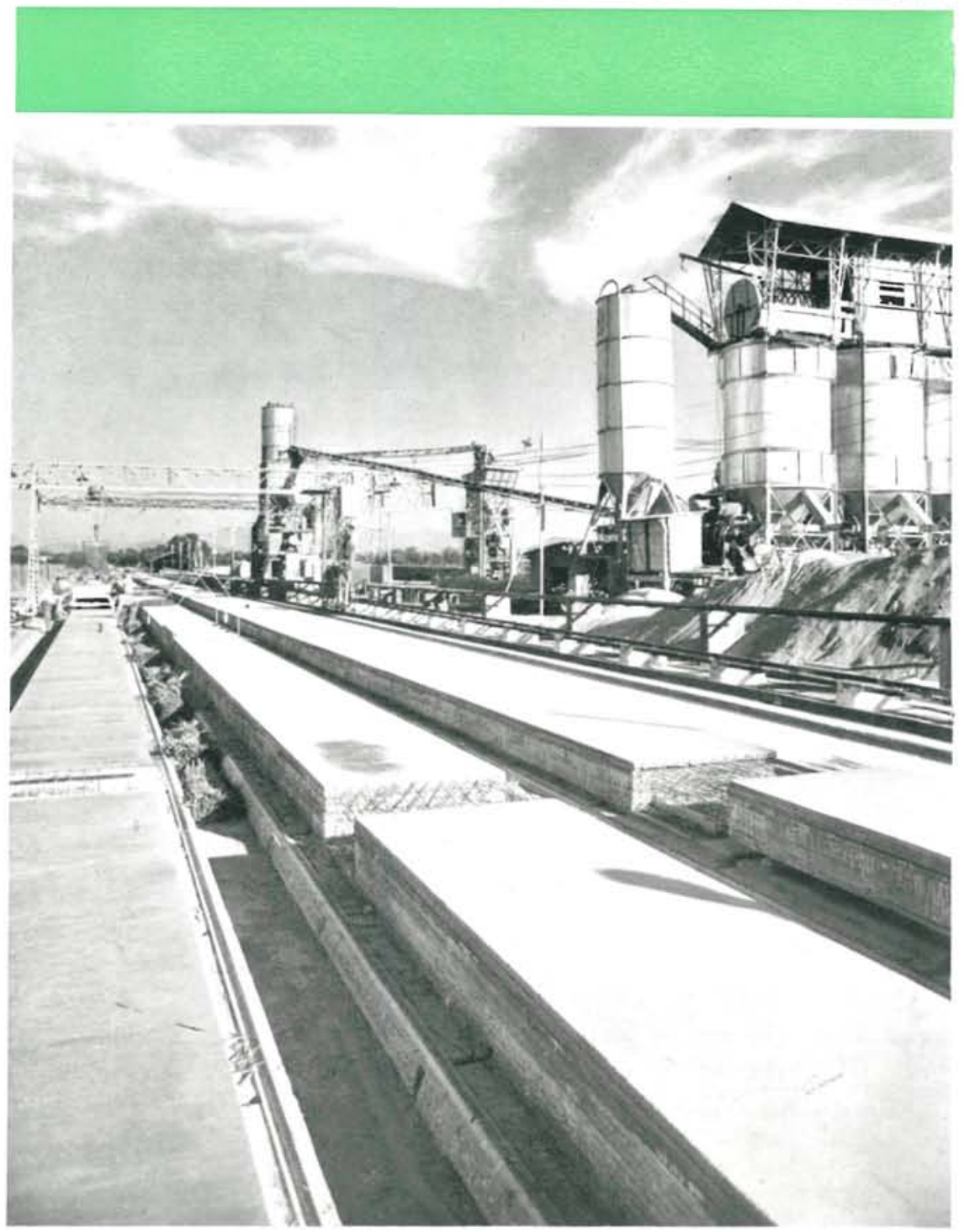


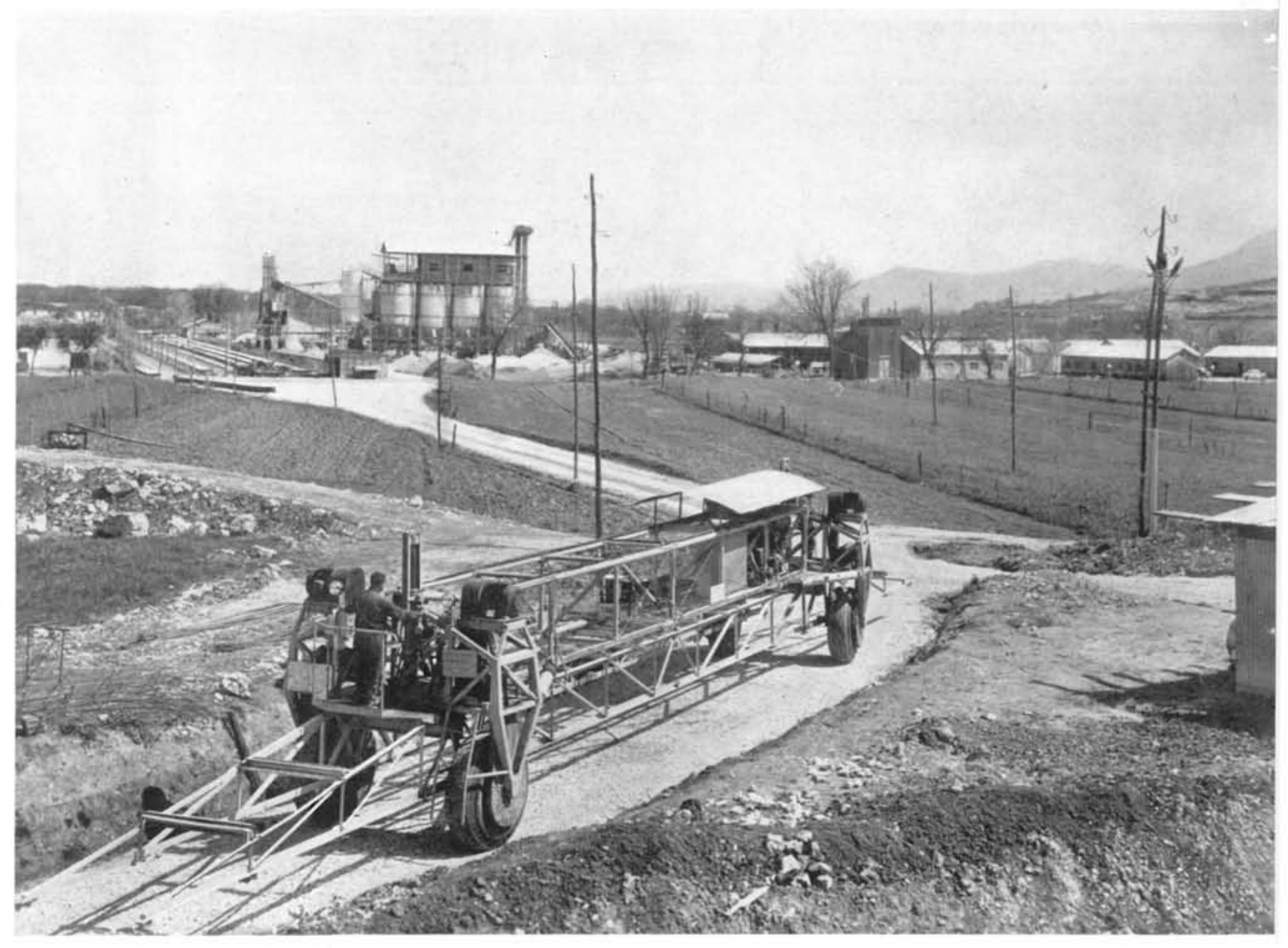

Plataforma para el transporte de loses.

4) La dimensión preponderante, que es la correspondiente a la dirección del pretensado, será lo más amplia posible (se han prefabricado losas de hasta $30 \mathrm{~m}$ ), y, además, la que mejor se adapte a amplias variaciones de la deformación.

5) La otra dimensión será función del espesor de la losa.

6) La precompresión longitudinal con armaduras de alambres adherentes, pretensados, densamente dispuestos y de pequeño diámetro.

7) Armaduras de repartición formando un ligero reticulado, soldado eléctricamente, denso y de reducido peso.

Los primeros ensayos han sido satisfactorios. Por ejemplo: una losa de $3 \mathrm{~cm}$ de espesor se dejó curvar, antes de romperse, hasta un radio de $1,50 \mathrm{~m}$. Se trataba de dar solución a problemas técnicos, pero preferentemente de poner en práctica la ejecución a escala industrial de estas losas.

En colaboración con la sociedad Precem, de Verona, se han podido poner a punto todos estos problemas, a los que se han unido los de perfeccionamiento del sistema de construcción aplicado a un tramo de canal que ha servido de espécimen experimental. En estos ensayos se han probado varios tipos de juntas, que llevaron a la conclusićn de que el tipo más adaptable es aquel que tiene en el borde de la losa un acanalado, que se sella con materiales de cemento expansivo. 



Prerrevestimiento con bloques de hormigón poroso drenante para el canal de Pontecorvo.

Ventosas para el aparejo de elevación de losas.

Las características generales de estas losas de hormigón armado pretensado se pueden resumir como sigue:

- Prefabricación a escala industrial.

- Grandes dimensiones.

- Gran flexibilidad y posibilidad de adaptación plástica.

- Impermeabilización.

-Duración.

- Economía máxima de materiales, pero contando con un taller de prefabricación especializado en alto grado y disponiendo de medios adecuados para el transporte y colocación de elementos en obra.

Estos sistemas de revestimiento han sido propuestos a una empresa de explotaciones hidroeléctricas en el valle del $\mathrm{Po}$ donde se puede observar un ejemplo del máximo relieve en la aplicación práctica de estos revestimien. tos. 


\section{Realizaciones}

Revestimiento de los taludes de un canal de derivación de la Sociedad Romana de Electrificación durante el período 1958-1959.

El canal de Pontecorvo, parcialmente en trinchera y terraplén, de unos $9 \mathrm{~km}$ de longitud, de sección transversal en forma parabólica, de unos $20 \mathrm{~m}$ de anchura y $6,5 \mathrm{~m}$ de altura o pro fundidad, ha sido objeto de aplicación de este tipo de revestimiento.

Este canal atraviesa terrenos de diferente naturaleza-fangosos, arcillosos, a veces en roca - y toda la parte en trinchera se halla sometida a la influencia de una capa freática, alta, que interesa gran parte de la sección del canal.

Teniendo en cuenta las consideraciones an. teriormente expuestas, la solución adoptada ha sido la siguiente:

El terreno excavado se consolidó moderadamente, precaución que se extendió a las zonas en terraplén, en las que la compactación fue más severa.

Sobre el terreno compactado se extendió una capa de piedra, drenante, de materiales calcáreos de granulometría discontinua y de $10 \mathrm{~cm}$

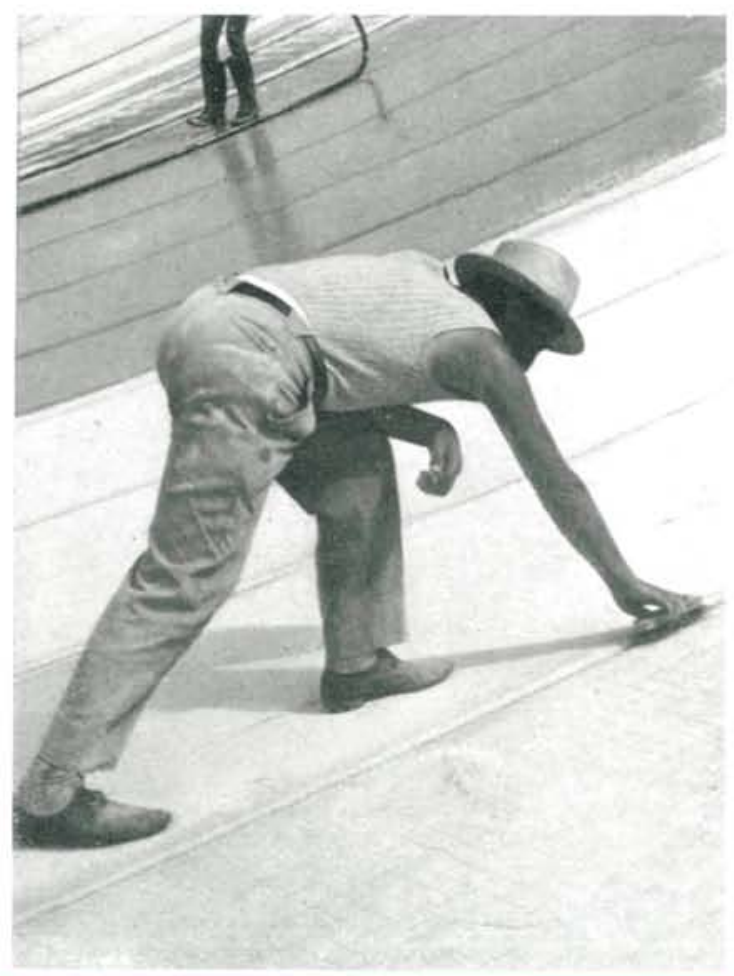

Preparación de juntas antes de sellar con «Emboco».

Colocación de losas utilizando ventosas, en el valle del Po

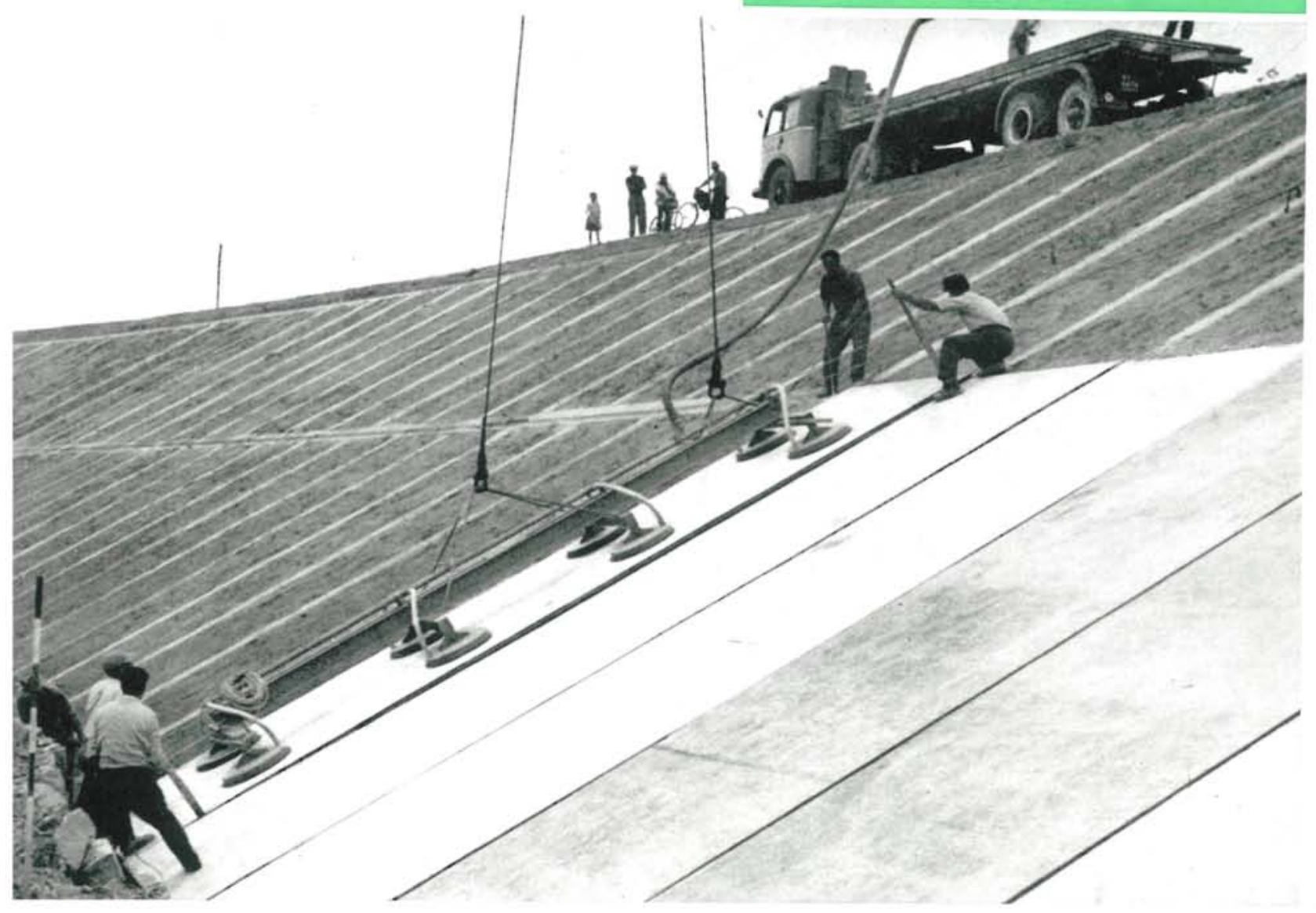



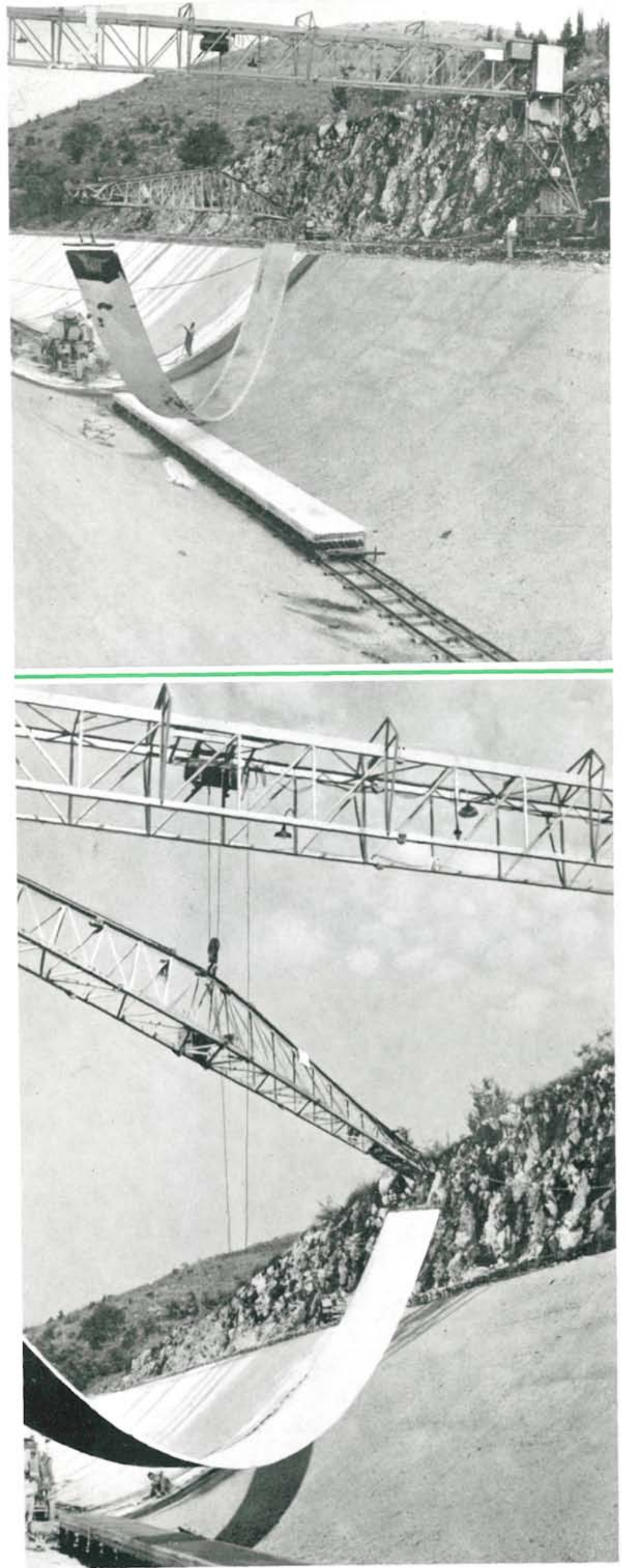

Traslación de una losa flexible de hormigón pre tensado.

Colocación de una losa.

de espesor; además, y como alternativa, se podian colocar bloques prefabricados, huecos, de hormigón poroso. La función de este revestimiento es la de drenar los taludes y recoger el agua dentro de una cuneta prevista en el fondo del canal.

El prerrevestimiento se enrasó con una lechada de cemento que actuaba como capa de sellado y de regularización del lecho de apoyo de la pavimentación con losas ligeras de hormigón armado pretensado.

Las losas que constituyen la pavimentación impermeable del canal se han prefabricado en un taller a tal fin instalado en la proximidad de la obra. El banco de prefabricación tenía una longitud de $250 \mathrm{~m}$ y el hormigonado se realizó con 10 a 12 tongadas de hormigón colocado mediante maquinaria especial vibro-acabadora, con vibración de alta frecuencia. Las losas tenían $3 \mathrm{~cm}$ de espesor, $1,25 \mathrm{~m}$ de anchura y de 25 a $30 \mathrm{~m}$ de longitud.

Después de endurecer y de tesar las armaduras, las losas se levantaban del banco y colocaban sobre mesillas con ruedas neumáticas para su transporte hasta el canal, donde se llevaban al lugar de colocación mediante trenes sobre vía «decauville» que se movían en el fondo del canal.

Una vez las losas en el lugar de ubi. cación, se amarraba cada una de ellas por sus dos extremidades a un puente-grúa instalado sobre las banquetas y provisto de un balancín giratorio para la maniobra de elevación. Se elevaban dichas losas y se les hacía formar una catenaria, dada su gran flexibilidad, colocándolas al fin, una al lado de otra, sobre una tongada de mortero fresco, apenas rociado y en posición según las generatrices del canal. La flexibilidad de las losas no permitía se adaptasen a la forma parabólica del perfil del canal, por lo que se les aplicaba un rodillo vibrante, de neumáticos, que recorría las losas inmediatamente después de haberlas colocado, facilitando asi la aproximación hacia la tongada de mortero, todavia fresca.

La última operación consistía en unir, en su parte superior, las losas, hormigonando los alambres sobresalientes, para después proceder al rejuntado de juntas transversales con un mortero especial, llamado Emboco, que consiste en una masa de cemento, arena, granulado metálico y agua, formando un conjunto ex. pansivo.

E1 revestimiento -- anteriormente expuesto-se comporta como si se tratase de una yuxtaposición de simples losas prefabricadas, de hormigón pretensado, rígidas, aun contando con las juntas, pues conservan cierta elasticidad y plasticidad suficiente para excluir cualquier posibilidad de fisuración superficial o dislocación. 

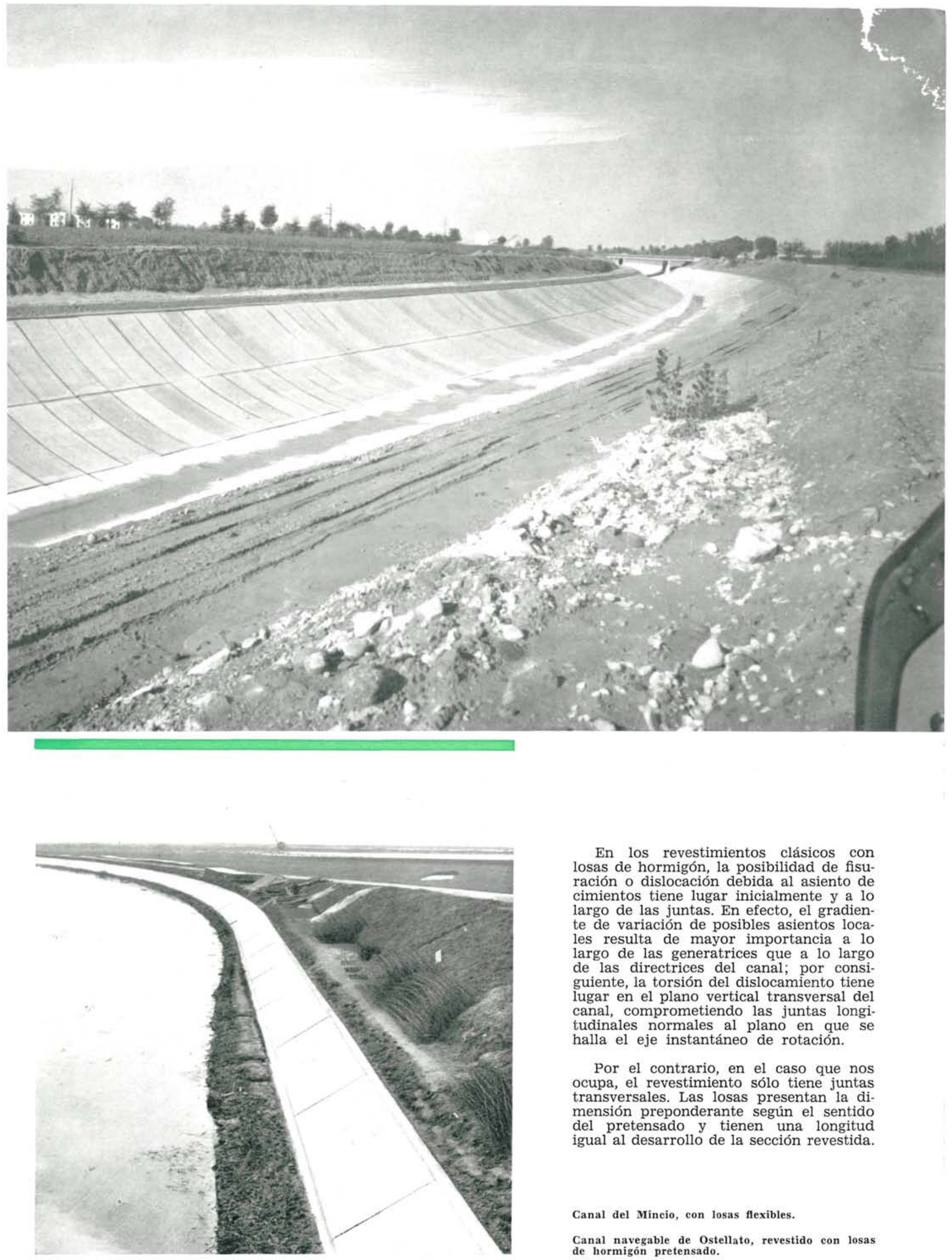

En los revestimientos clásicos con losas de hormigón, la posibilidad de fisuración o dislocación debida al asiento de cimientos tiene lugar inicialmente y a lo largo de las juntas. En efecto, el gradiente de variación de posibles asientos locales resulta de mayor importancia a lo largo de las generatrices que a lo largo de las directrices del canal; por consiguiente, la torsión del dislocamiento tiene lugar en el plano vertical transversal del canal, comprometiendo las juntas longitudinales normales al plano en que se halla el eje instantáneo de rotación.

Por el contrario, en el caso que nos ocupa, el revestimiento sólo tiene juntas transversales. Las losas presentan la dimensión preponderante según el sentido del pretensado y tienen una longitud igual al desarrollo de la sección revestida.

Canal del Mincio, con losas flexibles.

Canal navegable de Ostellato, revestido con losas de hormigón pretensado. 


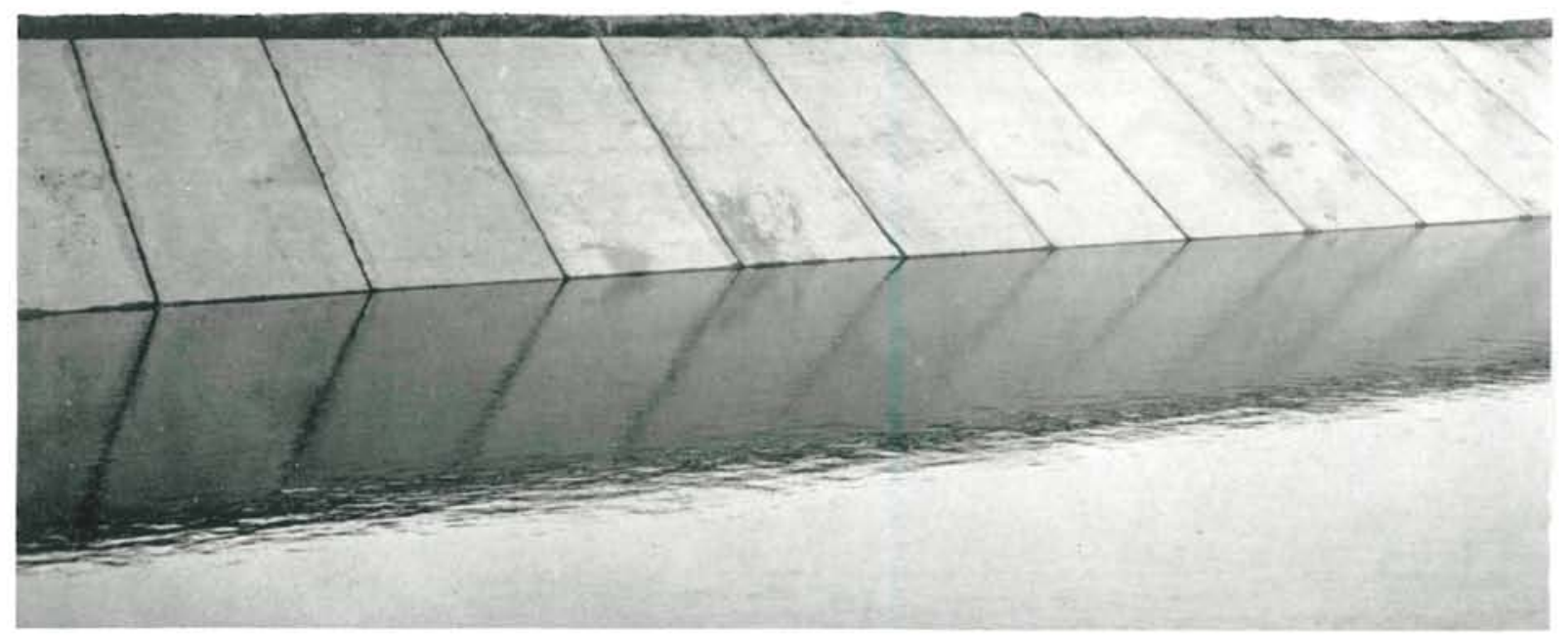

Canal del Po sin plena carga.
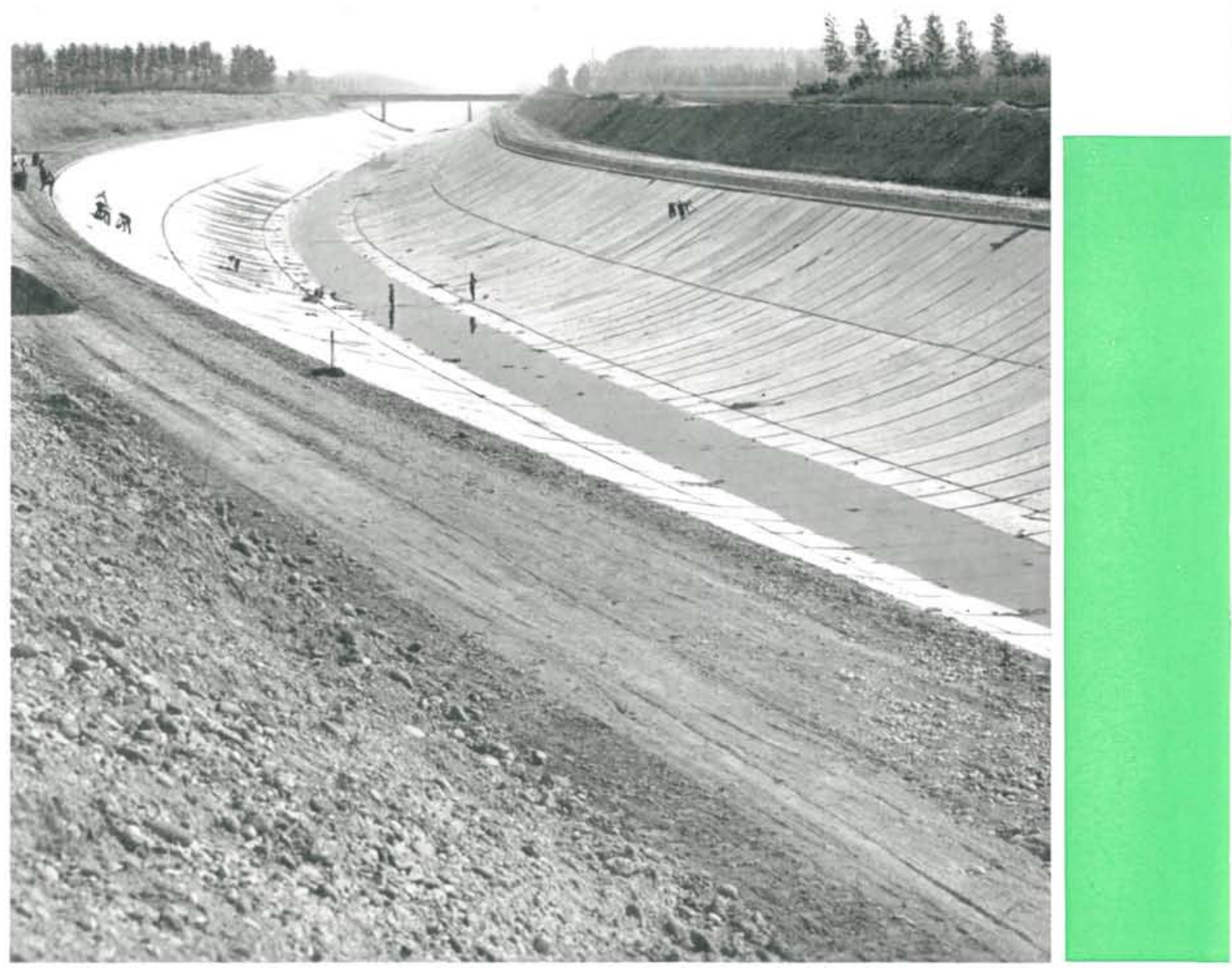

Terminado el revestimiento se procede al rejuntado. 


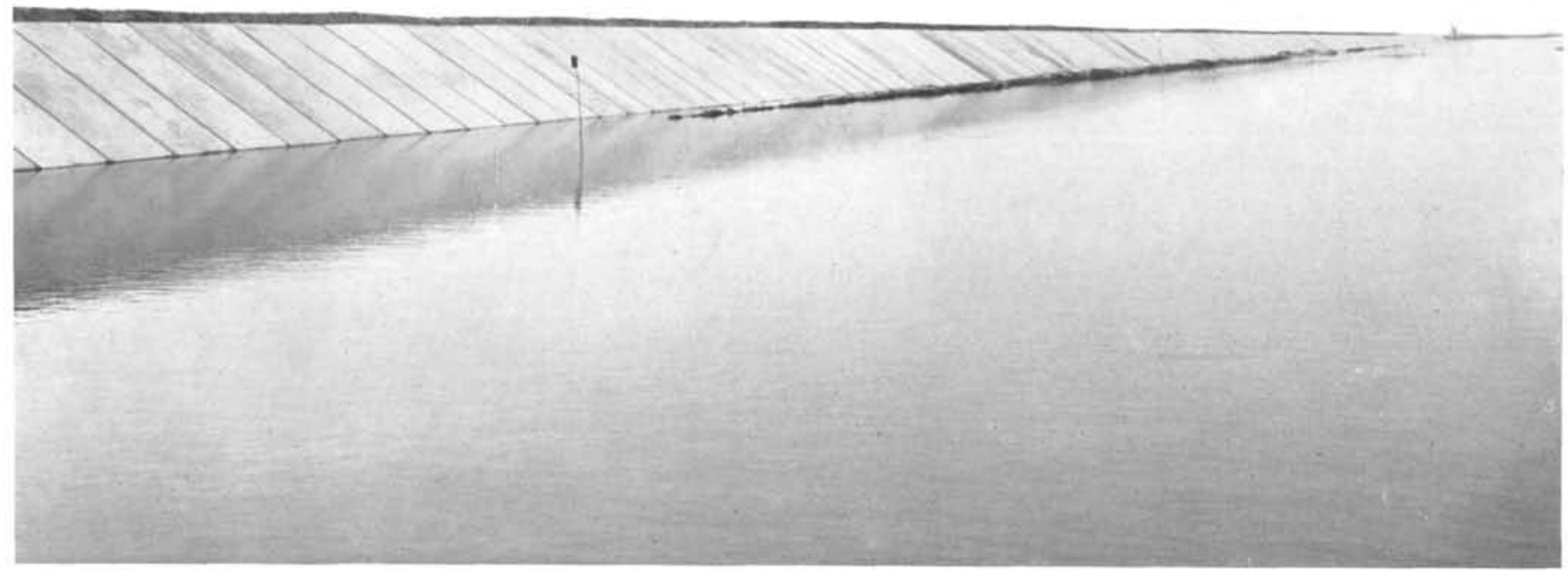

En lo que respecta a los efectos térmicos e higroscópicos, el revestimiento se comporta satisfactoriamente. En el sentido longitudinal se ejecuta una interrupción en las juntas «articuladas», espaciadas a 1,25 m. En el sentido transversal actúa la precompresión del hormigón y, además, un incremento automático de compresión a lo largo del perímetro mojado debido a la flexión inducida por la curvatura.

El procedimiento adoptado en el canal Pontecorvo ha experimentado una meticulosa puesta a punto, ya que se trataba de actuar mecánicamente tanto en lo que respecta a la fase de prefabricación como a la de colocación, ayudándose de instrumentos de gran precisión. En compensación, el trabajo se ha desarrollado muy rápidamente, colocando $300.000 \mathrm{~m}^{2}$ de losas en menos de seis meses, con mano de obra especializada, pero en número reducido.

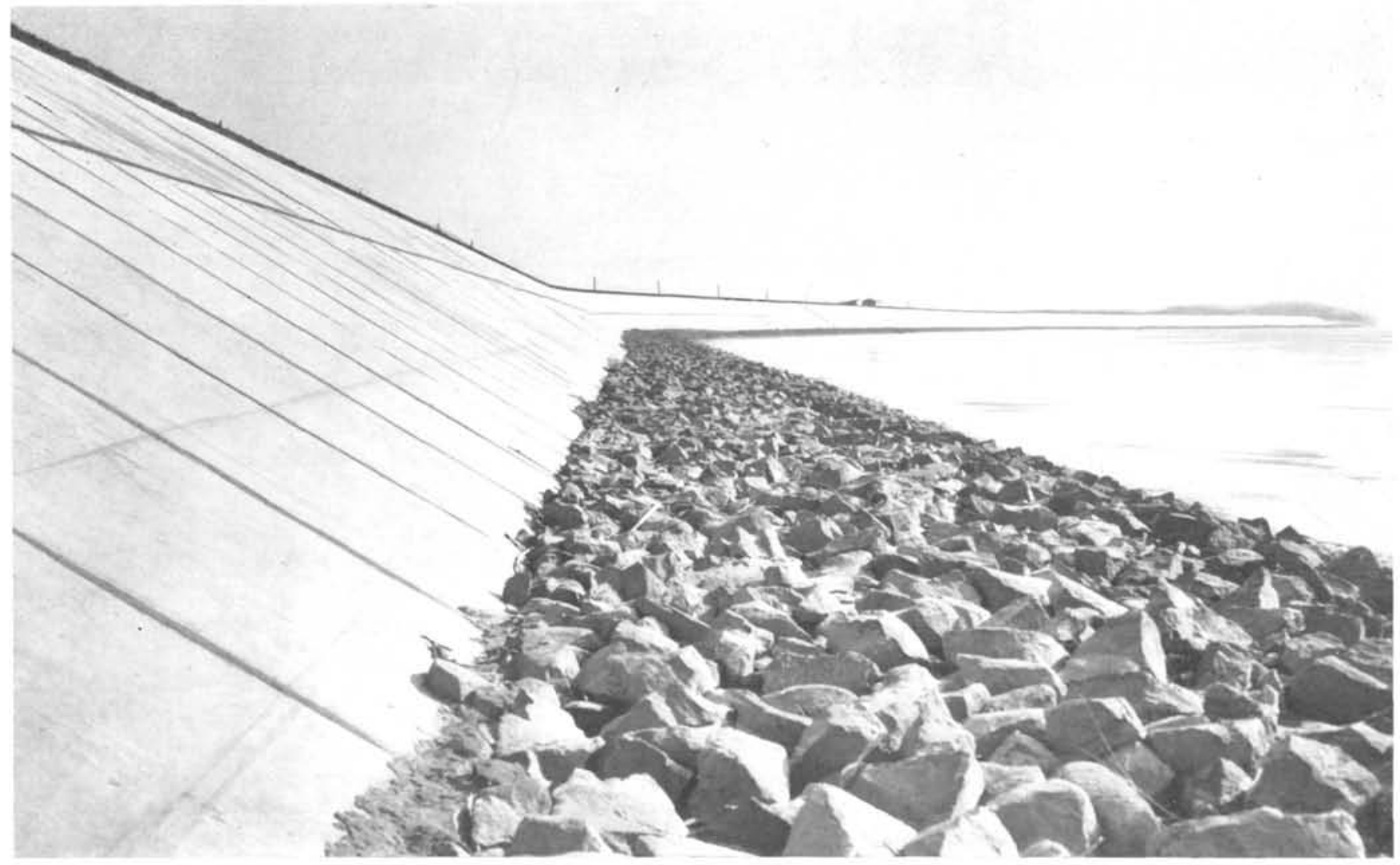

Juntas selladas en el canal del Po. 


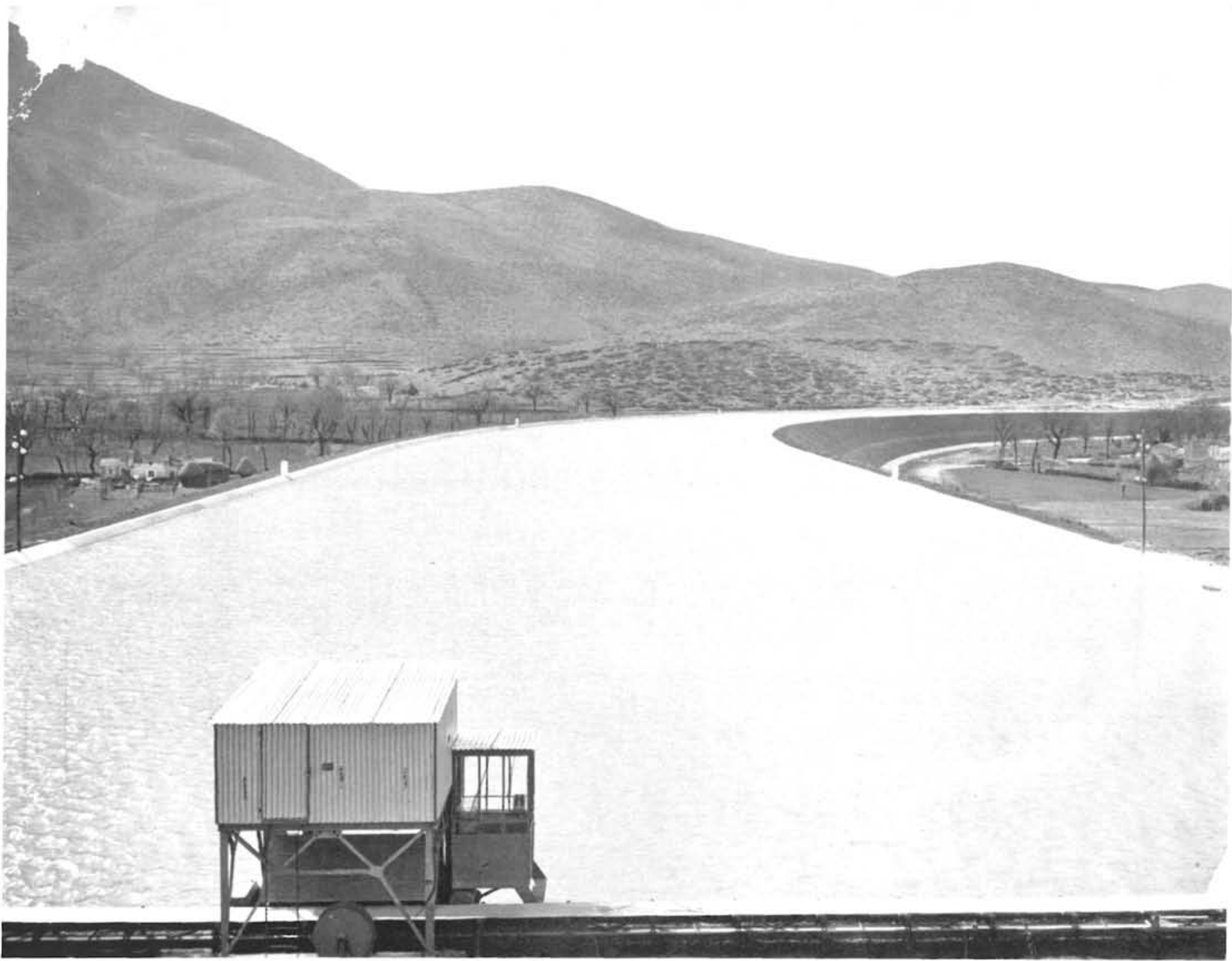

Canal de Pontecorvo, con aguas máximas.

Hasta el momento, el canal en servicio, sometido a carga continua, viene funcionando satisfactoriamente, permaneciendo impermeable el revestimiento.

Entre otras aplicaciones, muy similares en lo que a revestimientos con losas de hormigón pretensado se re. fiere, podemos citar los diques revestidos en el valle del río Po en Bergantino, el canal navegable de Ostellato-Migilarino, el canal de derivación del Mincio en Mantova y otras obras de este género.

\section{Comalensiones}

Hasta la actualidad se han colocado en obra más de un millón de losas de hormigón pretensado de este tipo, sin que los revestimientos experimenten desperfectos, por lo que su comportamiento ha sido satisfactorio desde el punto de vista de eficiencia y economía.

El éxito se debe compartir con la concepción moderna en el arte de construir actualmente apoyado en:

a) Prefabricación en talleres calificados.

b) Producción al ritmo industrial.

c) Productos de alta calidad.

d) Mecanización, medios de transporte y maquinaria auxiliar.

e) Personal reducido y especializado.

El empleo de materiales nobles y la manipulación apropiada permiten la reducción cuantitativa. La econo. mía del procedimiento depende, en gran manera, de la posibilidad de empleo en gran escala. 


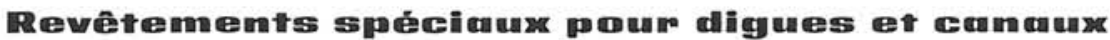

Silvano Zorsi, ingénieur.

Sont résumés ici les possibilités et les avantages qu'offrent les revêtements spéciaux de digues et de canaux et, en général, de tous les grands ouvrages hydrauliques, pour lesquels l'étanchéité est d'importance primordiale, puisqu'il s'agit de conserver las débits initiaux ou de retenue d'eaux, selon les cas.

Les progrès des techniques modernes, en particulier la préfabrication, ont permis ce type de revêtements à l'aide de dalles de grandes longueurs, extrèmement fiexibles et étanches, en béton armé et précontraint.

Bien que les possibilités et les types de revêtement soient nombreux, celui que nous présentons est remarquable: il se com. pose de plaques ou de dalles étroites, très flexibles, placées transversalement selon l'axe du canal sur des couches de mortier de ciment et dont les joints sont scellés, finalement, avec un mortier spécial appelé «Emboco».

L'exposé théorique de la méthode est complété par une série de réalisations, actuellement en service et donnant toutes satisfactions.

Ce travail se termine par quelques recommandations simples et brèves qui constituent, par elles-mêmes, les conclusions que l'on peut tirer de la méthode pour sa bonne exploitation et son plein succès, tant du point de vue technique qu'économique.

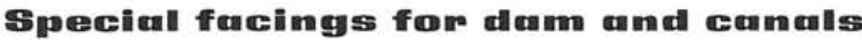

Silvano Zorsi, engineer.

This paper summarises the possibilities and advantages of adopting special facings for dykes and canals, and generally for all large hydraulic works, where waterproofing is of paramount importance.

Technical advances in recent years have made it possible to prefabricate many facing units used in these types of works; large slabs, made in reinforced and prestressed concrete, are flexible, and watertight.

Although there are many possibilities and types of facing treatments, the one described here is outstanding. It consists of narrow slabs, highly flexible, placed tranversally with respect to the canal axis. They are attached with cement of narrow slabs, highly flexible, placed tranversally with respect to the canal axis.
mortar, and the joints are finally sealed with a special mortar, called «Emboco».

In this article a description is also given of a series of projects, where facing treatments have been used, which are now successfully in service.

Finally some general and simple advise is given on the best procedure to apply these facing treatments in the most effective and economic manner.

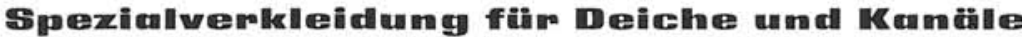

Silvano Zorzi, Ingenieur.

In dieser Arbeit werden Möglichkeiten und Vorteile von Spezialverkleidungen für Deiche, Kanalabhänge und Wasserbaukonstruktionen im allgemeinen aufgeführt, bei denen eine einwandfreie Isolierung von grosser Bedeutung für dar Stauen
von Wassermengen ist.

Dureh den Fortsehritt der modernen Technik, vor allem die Vorfabrikation, ist es möglich geworden, als Verkleidungs material lange, äusserst flexible und wasserdichte Platten aus Stahl- und Spannbeton zu verwenden.

Obwohl es zahlreiche Möglichkeiten und Typen dieser Verkleidung gibt, hebt sich die nachstehend beschriebene beson. ders hervor: Sie besteht aus schmalen, äusserst flexiblen Platten, die quer zur Achse des Kanals auf eine Zementmörtelschicht gelegt werden. Die entstandenen Fugen werden danach mit einem Spezialmörtel «Emboco» verkittet.

Zur Ergänzung der theoretischen Ausführungen werden noch eine Reihe von Beispielen genannt, die in der Praxis schon zufriedenstellende Ergebnisse gezeitigt haben.

Die Arbeit schliesst mit einer Reihe von einfachen und kurzen Empfehlungen, die eine Art Schlussfolgerung für eine erfolgreiche und bessere Ausnützung dieser Verkleidungsart sowohl vom technischen als auch vom finanziellen Standpunkt darstellen. 